\title{
Diabetes mellitus asociada al uso crónico de antipsicóticos atípicos
}

\author{
Álvaro Argoty Vallejos Narváez a, Giovanny Armando Chamorro b,c \\ Laura Melissa Rodríguez López ${ }^{\text {b,c }}$
}

\begin{abstract}
${ }^{a}$ Médico Especialista en Epidemiología, Magíster en Farmacología, Magíster en Educación para profesionales de la salud, Doctorando en Ciencias de la Salud, Jefe Cátedra de Farmacología, Facultad de Medicina. Fundación Universitaria Ciencias de la Salud. ORCID: https://orcid.org/0000-0003-1181-5013, avallejos@, fucsalud.edu.co; ${ }^{b}$ Médico residente especialización en psiquiatría, Fundación Universitaria Ciencias de la Salud. ORCID: https://orcid.org/0000-0002-7457-8695 , gaargoty@fucsalud.edu.co ; ${ }^{\circ}$ Médica residente especialización en psiquiatría, Fundación Universitaria Ciencias de la Salud. ORCID: https://orcid.org/00000003-0266-1147,1mrodriguez3@,fucsalud.edu.co DOI: https://doi.org/10.22517/25395203.24439
\end{abstract}

\section{Resumen}

Los agentes antipsicóticos son considerados como el principal tratamiento farmacológico para el manejo de mantenimiento y el tratamiento de casos agudos de la esquizofrenia y otros trastornos mentales. Estos medicamentos están clasificados en dos grupos: los antipsicóticos de primera generación o antipsicóticos típicos (AT) y los antipsicóticos de segunda generación o antipsicóticos atípicos (AA). En relación con el uso de los antipsicóticos típicos (AT), se han descrito importantes efectos adversos, sobre todo síntomas extrapiramidales (EPS), lo cual ha relegado su uso y fomenta el mayor empleo en la práctica clínica de los antipsicóticos atípicos (AA) gracias a la adecuada efectividad clínica demostrada hasta el momento. Sin embargo, estos medicamentos están asociados con diferentes alteraciones metabólicas, entre las cuales se encuentra un mayor riesgo de diabetes; para lo cual se han propuesto varios mecanismos etiopatogénicos. En esta revisión narrativa, se presenta un resumen actualizado de la literatura de los últimos 5 años sobre el uso de antipsicóticos atípicos y la diabetes mellitus tipo 2, haciendo énfasis en las diferentes hipótesis reportadas hasta el momento que involucran el aumento de peso y diferentes efectos mediados por hormonas, receptores de neurotransmisores y neuropéptidos que impactan en la génesis de los estados hiperglucémicos y de insulinorresistencia.

Palabras clave: antipsicóticos; diabetes mellitus tipo 2; efectos adversos a largo plazo; reacciones adversas

\section{Diabetes mellitus association with chronic use of atypical antipsychotics}

\begin{abstract}
Antipsychotic agents are currently considered as the main pharmacological treatment that helps the maintenance management of acute schizophrenia and other mental disorders. These drugs are classified into two groups: First-generation antipsychotics (FGAs) or typical antipsychotics, and Second-generation antipsychotics (SGAs) also known as atypical antipsychotics (AAP). In relation to the use of typical antipsychotics, some relevant adverse effects have been described, especially in terms of extrapyramidal side effects (EPS), which has downgraded their use and encourages the treatment of atypical antipsychotics (AAP) due to the adequate clinical effectiveness demonstrated so far in the practice. However, these drugs have been associated with different metabolic alterations such as the increased risk of suffering from diabetes; thus, several etiopathogenic mechanisms have been proposed. In this narrative review, we seek to present an updated summary of the literature of the last five years about the use of atypical antipsychotics and type 2 diabetes mellitus. Here it has been emphasized the different hypotheses reported so far that involve weight gain and different effects mediated by hormones, neurotransmitter receptors, and neuropeptides that impact on the genesis of hyperglycemia and insulin resistance states.
\end{abstract}

Keywords: Antipsychotic agents; type 2 diabetes mellitus; long term adverse effects; Drug-Related Side Effects and Adverse Reactions

\section{Introducción}

Los fármacos antipsicóticos son el pilar fundamental en el tratamiento agudo y de mantenimiento de la esquizofrenia y otros trastornos mentales, donde se incluyen los trastornos bipolares, las demencias y la irritabilidad relacionada con el autismo $(1,2)$.

El primer antipsicótico, lanzado en 1952, fue la clorpromazina; medicamento con gran éxito para el tratamiento de esquizofrenia, por lo cual, posteriormente se desarrollaron compuestos relacionados que actualmente se conocen como antipsicóticos de primera generación o antipsicóticos típicos (AT) $(1,2)$. 
Sin embargo, muchos pacientes tratados con AT desarrollaron efectos extrapiramidales secundarios (EPS) y trastornos graves del movimiento; lo que llevó a la introducción en 1990 de agentes que disminuyeran los efectos extrapiramidales, pero que conservaran su efecto antipsicótico; conocidos como antipsicóticos atípicos (AA) o de segunda generación. Entre los AA más importantes y utilizados en la práctica clínica se encuentran la clozapina, que fue el primer agente perteneciente a este grupo, seguida por olanzapina, quetiapina, risperidona, aripiprazol, paliperidona, entre otros. Ahora bien, la disminución sustancial de EPS afectó el efecto metabólico de los AA sobre el organismo, generando diversos trastornos, entre los cuales se encuentra el desarrollo de diabetes mellitus tipo 2 (DMT2) $(1,3)$

El propósito de este documento es realizar una revisión actualizada de la literatura sobre la fisiopatología de DMT2 asociadas al uso crónico de los antipsicóticos atípicos, con el fin de comprender mejor los factores relacionados. Para ello, se realizaron búsquedas en MEDLINE / PUBMED utilizando los términos "antipsicóticos"; "diabetes mellitus tipo 2" y "efectos secundarios relacionados con las drogas y reacciones adversas" en artículos publicados desde el año 2015 en adelante, fechas anteriores no se consideraron, salvo que se tratasen de textos relevantes.

\section{Fisiopatología del riesgo de DM2 por antipsicóticos atípicos}

La prevalencia de diabetes mellitus tipo 2 entre las personas que se encuentran en tratamiento con antipsicóticos es de aproximadamente 10\%, es decir, 2-3 veces más alta que en la población general (4).

Se han propuesto diferentes hipótesis mediante las cuales se intenta explicar el mecanismo fisiopatológico de la asociación entre el uso de medicación antipsicótica y DMT2, en algunos casos establecida como consecuencia del aumento de peso y síndrome metabólico; sin embargo, esta revisión se centra en aquellos mecanismos directos sobre el metabolismo de la glucosa (5). Entre ellos se puede enunciar: a) aumento de peso por resistencia a la insulina inducida por medicamentos antipsicóticos; b) mecanismos de acción de antipsicóticos atípicos relacionados con generación de DMT2 c) efecto directo en la señalización de la insulina y d) efecto citotóxico en las células beta del páncreas inducido por el mecanismo de acción (5). A continuación, se explicará cada una de estos mecanismos:

\section{A. Aumento de peso dependiente de resistencia de insulina inducida por antipsicóticos atípicos.}

La tasa de obesidad en personas con enfermedad mental grave aumenta de 2 - 3 veces en comparación con la población general, se ha reportado que cada $1 \mathrm{~kg} / \mathrm{m} 2$ en IMC aumenta el riesgo de DMT2 en un $8.4 \%(3,4)$. Se conoce a los antipsicóticos atípicos como "obesogénicos" ya que los altos niveles de tejido adiposo están fuertemente relacionados con resistencia a la insulina; a menudo se evidencia el aumento de peso dentro de las primeras 8 semanas, siendo más evidente en población que no ha recibido previamente medicación antipsicótica, mujeres y pacientes jóvenes $(1,3)$.

Hallazgos que se describen en diferentes estudios, por ejemplo, Sjo y col. en una cohorte retrospectiva en pacientes jóvenes, demostró que el uso de AA por primera vez, se asoció a un mayor aumento de peso (6). En la misma línea de investigación, Galling y col., realizaron un metaanálisis que demostró que a pesar de la baja incidencia de DMT2 en pacientes jóvenes, expuestos a antipsicóticos atípicos, el riesgo acumulado de DMT2 fue significativamente mayor (7). Por otra parte, Ingimarsson y col. resaltó que el uso de clozapina se asoció con un mayor riesgo de desarrollar DMT2 en mujeres que en hombres (8).

En relación con el efecto de los AA sobre la sensibilidad a la insulina y el aumento de peso, Burghardt y col. en un metaanálisis, revisó el efecto en voluntarios sanos; proporcionando evidencia preliminar de que estos efectos, pueden estar asociados con la duración del tratamiento y el tipo de antipsicótico atípico usado, es decir, se producen de forma independiente a la enfermedad psiquiátrica (9).

Entre los antipsicóticos atípicos se han descrito diferentes perfiles de riesgo metabólico, siendo valiosa por tanto la clasificación individual de cada medicamento asociado al riesgo diabetógeno $(1,10)$. En la figura 1 se presenta el riesgo de estos fármacos:

Riesgo de diabetes tipo 2

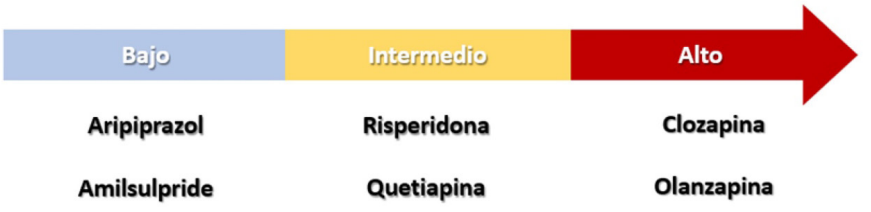

Figura 1. Clasificación de algunos antipsicóticos atípicos según el riesgo diabetógeno. 
Además, se ha descrito que dosis antipsicóticas altas se asocian con mayor riesgo de diabetes (4). Como lo demuestra Ulcickas y col., que tras el seguimiento de 2.5 años, a población expuesta a antipsicóticos atípicos, presentó un mayor riesgo de DMT2 para olanzapina, risperidona y quetiapina, con una posible relación dosisrespuesta (11).

\section{B. Mecanismos de acción de antipsicóticos atípicos relacionados con generación de DMT2}

Los antipsicóticos atípicos son antagonistas del receptor de dopamina D2, con la excepción del aripiprazol, que es un agonista parcial de D2; también ejercen su acción sobre una amplia gama de receptores que incluyen dopamina (D1R y D4R), serotonina (5-HT1A, 5-HT2A, 5-HT2C, 5-HT6, 5-HT7), histamina (H1R) y receptores muscarínicos (M3R) $(1,2)$.

Es importante tener en cuenta que los mecanismos farmacológicos implicados en el desarrollo de diabetes inducida por antipsicóticos atípicos aún continúa en investigación $(1,5)$. Sin embargo, dentro de las alteraciones metabólicas asociadas al uso de AA, se encuentran efectos mediados por diferentes hormonas, receptores de neurotransmisores y neuropéptidos (5); con el fin de comprender este proceso, a continuación presentamos las más influyentes:

\section{Hormonas}

a. Leptina: se relaciona con los niveles de adiposidad y aumento del apetito en pacientes en manejo con antipsicóticos. Es principalmente secretada por los adipocitos y está involucrada en el control de la ingesta de comida por su acción hipotalámica, es conocida como una de las hormonas "anorexigénicas". Sus niveles elevados se han asociado con el desarrollo de DMT2 así como con sus complicaciones micro y macrovasculares $(5,12)$.

Estos efectos metabólicos son inducidos de forma rápida, en especial durante el tratamiento con olanzapina; se ha demostrado que tratamiento $10 \mathrm{mg} /$ día por 3 días, aumenta los niveles de leptina y triglicéridos, frecuentemente elevados en pacientes con DMT2 (13).

b. Prolactina: los niveles séricos de prolactina están inversamente asociados con el riesgo de DMT2 y se relacionan con la estimulación de la proliferación de células $\beta$ del páncreas, producción y secreción de insulina. Se ha visto relacionada con la regulación de la secreción de citoquinas; adicionalmente, puede suprimir la producción de adiponectina e IL-6 que condiciona la adipogénesis y la respuesta inflamatoria $(5,14)$.

La hiperprolactinemia es conocida por ser uno de los efectos adversos más relevantes de los AT, característica que los diferencia de los AA (5).

\section{Neurotransmisores}

a. Receptor de histamina H1 (H1R): este receptor es bloqueado por los AA, siendo Clozapina y Olanzapina los que tienen mayor afinidad. Se cree que está relacionado con la homeostasis y el placer de la alimentación (5).

Los últimos hallazgos apuntan a la regulación al alza del H1R, como el responsable, del aumento de peso y la inducción a una desregulación del metabolismo de glucosa, dado el deterioro de la señalización de la insulina, a nivel del músculo esquelético (1).

b. Receptor de serotonina 5-HT2c (HT2RC): se cataloga como "anorexigénico" y es antagonizado potentemente por Clozapina y Olanzapina (5). Además, se ha informado la asociación entre varios polimorfismos en HT2RC y un mayor riesgo de síndrome metabólico, donde el efecto antagonista sobre este receptor puede estar implicado en la alteración del metabolismo de la glucosa (1).

c. Receptores adrenérgicos $\beta 3$ y $\alpha 1$ : existe correlación entre el antagonismo de estos receptores y el aumento de peso mediado por antipsicóticos; aunque su afinidad es leve (3).

d. Receptor muscarínico M3 (M3R): es el principal receptor de acetilcolina responsable de potenciar la liberación de insulina dependiente de glucosa en las células $\beta$, por lo que su bloqueo por AA altera el metabolismo de la glucosa llevando a cuadros de hiperglicemia y DMT2 (15). Desde el punto de vista bioquímico, se relaciona con la despolarización de la 
membrana celular, que conlleva a un aumento de la exocitosis de insulina en las células beta del páncreas. Así mismo, se establece relación con la alteración de la señalización muscarínica en áreas cerebrales que regulan la secreción de insulina por medio de la inervación vagal del páncreas (16).

\section{Neuropéptidos}

a. Receptor de Melanocortina 4 y FNDC (Factor neurotrófico derivado del cerebro): algunos fármacos antipsicóticos aumentan su expresión en el hipocampo y corteza prefrontal; asociada con aumento de la ingesta y por ende aumento de peso (3). FNDC está involucrado en la regulación y metabolismo de glucosa y energía, por lo cual tiene una correlación positiva con las lipoproteínas plasmáticas. Sin embargo, también se ha documentado un papel citoprotector, previniendo el agotamiento de las células beta pancreáticas, manteniendo su organización celular histológica (17).

\section{Efecto directo en la señalización de la insulina.}

La insulina es una hormona clave en el metabolismo de la glucosa, esta es secretada por las células $\beta$ del páncreas y ejerce su acción a nivel celular, con el aumento de absorción de glucosa, en especial en el músculo esquelético, el tejido hepático, renal y adiposo (15).

La resistencia a la insulina se refiere a la situación en la que las células objetivo pierden respuesta a la estimulación de insulina, reduciendo la absorción de glucosa (15). Los mecanismos que producen esta resistencia a la insulina aún no se comprenden completamente y pueden ocurrir en diferentes niveles de la señalización de insulina (4) y son cambios que ocurren independientemente del aumento de peso. Normalmente, la insulina al unirse a su receptor genera la autofosforilación del mismo que a su vez activa los receptores de tirosina quinasas que fosforilan los sustratos del receptor de insulina 1 (IRS 1), lo que lleva a la activación de la vía de la fosfoinositida 3-quinasa (PI3K), que convierte fosfatidilinositol $(3,4)$-bisfosfato (PIP2) en fosfatidilinositol (3,4,5) -trifosfato (PIP3). El PIP3 se une a proteína quinasa $\mathrm{B}$ (también conocida como $\mathrm{Akt}$ ); este proceso conduce a la translocación de Akt a la membrana de la célula, donde es fosforilada y finalmente desencadena una cascada de señalización que provoca la translocación de GLUT4 a la membrana celular para el transporte de glucosa (15). Los antipsicóticos atípicos disminuyen la fosforilación de IRS-1 inducida por insulina e inhiben la actividad de Akt2 causando resistencia a la insulina (15). Por tanto, se afecta la vía de señalización de insulina en las células dependientes de glucosa, proceso que es importante para el desarrollo de DMT2. En la figura 2 se describe este proceso:

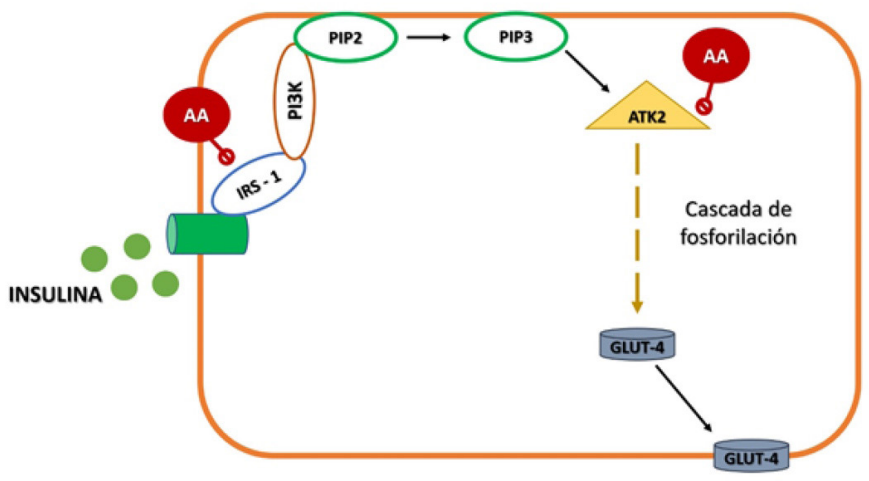

Figura 2. Vía de señalización de insulina y efecto de antipsicótico atípico.

\section{Efecto citotóxico en las células beta del páncreas}

Se ha reconocido el papel de la célula $\beta$ del páncreas en la patogénesis de DMT2 ya que la resistencia a la insulina per se, se considera insuficiente para causar diabetes (15). Por tanto, el impacto de los antipsicóticos atípicos sobre estas células permite el planteamiento del efecto diabetogénico de estos medicamentos (18).

Se han descrito diferentes mecanismos que explican la disfunción de la célula beta pancreática causada por antipsicóticos atípicos; entre estos podemos nombrar:

1. Los antipsicóticos bloquean los receptores D2 y D3 que además de encontrarse a nivel cerebral se encuentran en las células $\beta$ del páncreas. La dopamina está involucrada en la regulación de la glucosa; mediante feedback negativo, inhibe la liberación de insulina y la secreción crónica de la misma, que puede llevar a una resistencia similar a la hiperinsulinemia en DMT2; generando el agotamiento de las células $\beta$ del páncreas (3).

2. El bloqueo de la secreción de insulina también puede estar mediado por el antagonismo de los receptores: adrenérgico $\alpha 1$, serotoninérgico 5-HT2A y muscarínico M3R (15).

3. Los antipsicóticos atípicos también se han 
relacionado con un aumento en la apoptosis de las células $\beta$, lo que lleva a una hipotrofia pancreática con una consecuente disminución en la secreción de insulina. Proceso que puede estar mediado por la vía apoptótica mitocondrial (15).

\section{Otros efectos metabólicos de los antipsicóticos atípicos}

El aumento de peso inducido por antipsicóticos y la dislipidemia son efectos secundarios metabólicos que también son importantes tener en cuenta durante el tratamiento con AA (1).

La dislipidemia inducida por antipsicóticos atípicos suele manifestarse con un aumento del nivel de los triglicéridos, asociado a una disminución de lipoproteínas de alta densidad, que generalmente corresponde al estado metabólico inducido por estos medicamentos, donde se encontrarán altos niveles de glucosa, que a nivel hepático desequilibran el metabolismo de los lípidos (1).

Otro mecanismo implicado en la hiperlipidemia podría deberse a un efecto directo de los AA sobre el regulador principal de la biosíntesis de colesterol, independientemente de los cambios en el metabolismo de la glucosa $(1,19)$.

Finalmente, cabe resaltar que los pacientes con trastornos mentales graves tienen mayor probabilidad de desarrollo de cetoacidosis diabética $(\approx 10 \%)$ como primera manifestación de diabetes, cuando se compara con la población general $(4,15)$.

La ingesta de AA puede desencadenar cetoacidosis diabética por 1) complicación de diabetes tipo 1 preexistente, 2) DMT2 de novo inducida por AA (aumento de IMC y resistencia a insulina) o 3) cetoacidosis diabética fulminante imitando un cuadro de DMT2 por resistencia a la insulina inducida por AA de manera aguda, sin aumento de IMC (20).

\section{Aumento del riesgo cardiovascular con antipsicóticos atípicos}

Suele ser frecuente en personas con trastornos mentales graves encontrar factores de riesgo como una dieta poco saludable, tabaquismo, sedentarismo, aislamiento social y mecanismos específicos de la enfermedad que asociados al uso de antipsicóticos atípicos, generan un importante aumento de peso $(1,4)$.

Los efectos adversos demostrados de los antipsicóticos atípicos sobre el peso corporal, el metabolismo de glucosa y perfil lipídico pueden generar una mayor incidencia de enfermedad macrovascular, sin embargo, los estudios realizados hasta al momento no han demostrado mayor riesgo cardiovascular y mortalidad en pacientes que se encuentren en tratamiento con AA (4).

No obstante, es claro que el desarrollo de efectos secundarios a nivel del metabolismo de glucosa y lípidos pueden tener un impacto en la calidad de vida de los pacientes ya que estas alteraciones son puntos claves para la enfermedad cardiovascular y la muerte prematura (15).

Por tanto, se debe realizar un control adecuado de factores de riesgo cardiovascular en los diferentes controles de pacientes que toman (AA), con la toma de glucosa y perfil lipídico, así como el seguimiento del perímetro abdominal, dado que se ha estimado que entre el 20 y el $50 \%$ de los pacientes tratados son obesos y tienen diabetes $(1,15)$.

\section{Conclusiones}

Los antipsicóticos de segunda generación o antipsicóticos atípicos (AA) se desarrollaron con el objetivo de reducir los EPS y preservar la eficacia de su tratamiento, sin embargo, están asociados con alteraciones metabólicas graves.

Se puede concluir que el mecanismo común de desarrollo de diabetes inducido por AA es mediante la vía de aumento de apetito y consecuentemente de IMC que lleva a una elevación de la adiposidad abdominal con posterior desarrollo de resistencia a insulina y diabetes. Cabe mencionar que los sujetos que se encuentran en tratamiento con estos agentes, a menudo cursan con trastornos mentales como la esquizofrenia, en la cual hay presencia de síntomas negativos

que los inducen a llevar estilos de vida sedentarios que contribuyen a la agilización del proceso de aumento de peso y desarrollo de DMT2.

Los mecanismos farmacológicos implicados en el desarrollo de diabetes inducida por antipsicóticos atípicos continúan en investigación, si bien se ha demostrado la participación de diferentes hormonas, receptores y neuropéptidos que pueden interferir con los centros de saciedad, apetito y desregulación del metabolismo de glucosa. La alteración del metabolismo de glucosa mediado por AA puede estar asociada a el efecto directo en la vía de señalización de insulina y el daño sobre células beta del páncreas.

Las mujeres, población pediátrica y sujetos que no han recibido previamente medicación antipsicótica son más susceptibles a los efectos metabólicos de los antipsicóticos de segunda generación. 
Finalmente, es importante implementar medidas para prevenir, detectar y garantizar el diagnóstico temprano de diabetes en la población que usa antipsicóticos.

\section{Referencias}

1. Grajales D, Ferreira V, Valverde ÁM. SecondGeneration Antipsychotics and Dysregulation of Glucose Metabolism: Beyond Weight Gain. Cells. 2019;8(11):1336.

2. Taylor DM, Barnes TRE, Young AH. The Maudsley prescribing guidelines in psychiatry: John Wiley \& Sons; 2018.

3. Whicher CA, Price HC, Holt RIG. Mechanisms in endocrinology: Antipsychotic medication and type 2 diabetes and impaired glucose regulation. Eur. J.of Endocrinol. 2018;178(6):R245-R58.

4. Holt R. Association between antipsychotic medication use and diabetes. Curr. Diab. Rep. 2019;19(10):96.

5. Reynolds GP, Kirk SL. Metabolic side effects of antipsychotic drug treatment-pharmacological mechanisms. Pharmacology \& therapeutics. 2010;125(1):169-79.

6. Sjo CP, Stenstrøm AD, Bojesen AB, Frølich JS, Bilenberg N. Development of metabolic syndrome in drug-naive adolescents after 12 months of secondgeneration antipsychotic treatment. J. Child. Adolesc. Psychopharmacol. 2017;27(10):884-91.

7. Galling B, Roldán A, Nielsen RE, Nielsen J, Gerhard T, Carbon M, et al. Type 2 diabetes mellitus in youth exposed to antipsychotics: a systematic review and metaanalysis. JAMA psychiatry. 2016;73(3):247-59.

8. Ingimarsson O, MacCabe JH, Haraldsson M, Jónsdóttir $\mathrm{H}$, Sigurdsson E. Risk of diabetes and dyslipidemia during clozapine and other antipsychotic drug treatment of schizophrenia in Iceland. Nordic journal of psychiatry. 2017;71(7):496-502.

9. Burghardt KJ, Seyoum B, Mallisho A, Burghardt PR, Kowluru RA, Yi Z. Atypical antipsychotics, insulin resistance and weight; a meta-analysis of healthy volunteer studies. Progress in Neuro-Psychopharmacology and Biological Psychiatry. 2018;83:55-63.

10. Zhang Y, Liu Y, Su Y, You Y, Ma Y, Yang G, et al. The metabolic side effects of 12 antipsychotic drugs used for the treatment of schizophrenia on glucose: a network meta-analysis. BMC psychiatry. 2017;17(1):1-9.
11. Yood MU, DeLorenze GN, Quesenberry CP, Oliveria SA, Tsai A-L, Kim E, et al. Association between secondgeneration antipsychotics and newly diagnosed treated diabetes mellitus: does the effect differ by dose? BMC psychiatry. 2011;11(1):197.

12. Katsiki N, Mikhailidis DP, Banach M. Leptin, cardiovascular diseases and type 2 diabetes mellitus. Acta Pharmacologica Sinica. 2018;39(7):1176-88.

13. Albaugh VL, Singareddy R, Mauger D, Lynch CJ. A double blind, placebo-controlled, randomized crossover study of the acute metabolic effects of olanzapine in healthy volunteers. PLoS One. 2011;6(8):e22662.

14. Li J, Rice MS, Huang T, Hankinson SE, Clevenger $\mathrm{CV}, \mathrm{Hu} \mathrm{FB}$, et al. Circulating prolactin concentrations and risk of type 2 diabetes in US women. Diabetologia. 2018;61(12):2549-60.

15. Chen J, Huang X-F, Shao R, Chen C, Deng C. Molecular mechanisms of antipsychotic drug-induced diabetes. Frontiers in neuroscience. 2017;11:643.

16. Weston-Green K, Huang X-F, Deng C. Second generation antipsychotic-induced type 2 diabetes: a role for the muscarinic M3 receptor. CNS drugs. 2013;27(12):1069-80.

17. Bathina S, Das UN. Brain-derived neurotrophic factor and its clinical implications. Archives of medical science: AMS. 2015;11(6):1164.

18. Farino ZJ, Morgenstern TJ, Maffei A, Quick M, De Solis AJ, Wiriyasermkul P, et al. New roles for dopamine D 2 and D 3 receptors in pancreatic beta cell insulin secretion. Molecular psychiatry. 2019:1-16.

19. Canfrán-Duque A, Casado ME, Pastor Ó, SánchezWandelmer J, de la Peña G, Lerma M, et al. Atypical antipsychotics alter cholesterol and fatty acid metabolism in vitro. Journal of lipid research. 2013;54(2):310-24.

20. Vuk A, Rojnic Kuzman M, Baretic M, Matovinovic Osvatic M. Diabetic ketoacidosis associated with antipsychotic drugs: case reports and a review of literature. Psychiatria Danubina. 2017;29(2):121-35. 